\title{
ESTRATÉGIAS DE APRENDIZAGEM E A REGULAÇÃO DA EMOÇÃO NO ENSINO FUNDAMENTAL
}

\author{
Adriana Cristina Boulhoça Suehiro \\ Universidade Federal do Recôncavo da Bahia (UFRB) \\ Evely Boruchovitch \\ Universidade Estadual de Campinas (Unicamp) \\ Patrícia Waltz Schelini \\ Universidade Federal de São Carlos (UFSCar)
}

\begin{abstract}
Resumo
O presente estudo teve como objetivos caracterizar o uso de estratégias de aprendizagem e de regulação emocional de crianças brasileiras do Ensino Fundamental, examinar se existem diferenças nessas variáveis em razão do sexo, ano escolar e idade dos estudantes, bem como explorar possíveis relações entre elas. A amostra foi composta de 38 alunos, do Ensino Fundamental de escolas públicas. Os dados foram coletados por meio de duas escalas do tipo Likert. Não foram encontradas diferenças significativas entre meninos e meninas nas escalas de estratégias de aprendizagem e de regulação emocional. Os resultados revelaram que os estudantes de anos mais avançados obtiveram médias significativamente menores na escala de estratégias de aprendizagem quando comparados aos alunos que estão no meio do curso. As correlações tenderam a ser positivas e significativas entre os aspectos cognitivos, metacognitivos e a regulação emocional, todavia as magnitudes foram predominantemente fracas.

Palavras-chave: estratégias cognitivas; metacognição; emoções; educação básica.
\end{abstract}

\section{LEARNING STRATEGIES AND EMOTIONAL REGULATION IN ELEMENTARY SCHOOL}

\begin{abstract}
The aims of the present study were to characterize the use of learning and emotional regulation strategies of basic education Brazilian children, to examine whether there are differences in the major variables in relation to students' sex, school year, and age, as well as to explore possible relationship between the variables. The sample consisted of 38 basic education students from public schools. No significant gender differences emerged in the scores of learning strategies and emotional regulation scales. The results revealed that students who were more advanced in school had lower means in the learning strategies scale than those who were in the middle of the Basic Education. Correlations tended to be positive and significant between cognitive, metacognitive and emotional regulation, but their magnitudes were predominantly weak.
\end{abstract}

Keywords: cognitive strategies; metacognition, emotions, basic education. 


\title{
ESTRATEGIAS DE APRENDIZAJE Y AUTORREGULACIÓN EMOCIONAL EN LA ENSEÑANZA PRIMARIA
}

\begin{abstract}
Resumen
El presente estudio tuvo como objetivos caracterizar el uso de estrategias de aprendizaje y regulación emocional de niños brasileños de la Enseñanza Fundamental, examinar si existen diferencias en esas variables en razón del sexo, año escolar y edad, así como explorar posibles relaciones entre ellas. La muestra fue compuesta de 38 alumnos, de la Enseñanza Fundamental de escuelas públicas. Los datos fueron recolectados por medio de dos escalas del tipo Likert. No se encontraron diferencias significativas entre los niños y las niñas en las escalas de estrategias de aprendizaje y de regulación emocional. Los resultados revelaron que los estudiantes de años más avanzados obtuvieron promedios significativamente menores en la escala de estrategias de aprendizaje cuando comparados a los alumnos que están en el medio del curso. Las correlaciones tienden a ser positivas y significativas entre los aspectos cognitivos, metacognitivos y la regulación emocional, pero las magnitudes fueron predominantemente débiles.
\end{abstract}

Palabras clave: estrategias cognitivas, metacognición, emociones, enseñanza fundamental.

\section{INTRODUÇÃO}

Existem inúmeras evidências sobre os fatores que promovem a aprendizagem. Há congruência entre pesquisadores de que a aprendizagem de qualidade envolve a inter-relação de um conjunto de variáveis como o uso de estratégias de aprendizagem, a motivação adequada para aprender, o controle das emoções, entre outras (Linnenbrink-Garcia \& Pekrun, 2011; Mega, Ronconi, \& De Beni, 2013; Pressley, Borkowski, \& Schneider, 1989; Weinstein, Acee, \& Jung, 2011; Zimmerman \& Schunk, 2011). Essas características são também tidas como essenciais para a aprendizagem autorregulada, muita enfatizada nos dias de hoje e entendida como uma forma de aprender na qual o aluno se responsabiliza pelo seu próprio processo de aprendizagem (Linnenbrink-Garcia \& Pekrun, 2011; Mega et al., 2013; Pressley et al., 1989; Weinstein et al., 2011; Zimmerman \& Schunk, 2011). Assim, em linhas gerais, pode-se dizer que um estudante autorregulado é aquele que, com base no autoconhecimento, consegue empregar estratégias de aprendizagem de forma apropriada às demandas da tarefa, possui motivação autônoma ou intrínseca, bem como apresenta controle das suas emoções no que concerne aos desafios que emergem no contexto escolar (Linnenbrink-Garcia \& Pekrun, 2011; Mega et al., 2013; Pressley et al., 1989; Weinstein et al., 2011; Zimmerman \& Schunk, 2011).

As estratégias de aprendizagem são definidas como sequências de procedimentos ou atividades que os estudantes usam com o propósito de facilitar a aquisição, o armazenamento e/ou a utilização da informação (Nisbett \& Schucksmith, 1986). Em revisões extensas da literatura Boruchovitch (1999), Boruchovitch e Santos (2006), Boruchovitch, Schelini e Santos (2010) constaram que inúmeras são as classificações e taxionomias existentes para descrever as estratégias de aprendizagem (Dansereau et al., 1979; Dembo, 1994; Garner \& 
Alexander, 1989; Nisbett \& Schucksmith, 1986, Pozo, 1996; Weinstein \& Mayer, 1985; Weinstein et al., 2011; Zimmerman \& Martinez-Pons, 1986).

Como mencionado por Boruchovitch e Santos (2006), as várias nomenclaturas existentes acerca das estratégias de aprendizagem estão mais associadas aos diferentes termos empregados pelos pesquisadores do que a diferenças substanciais em seus significados. Nessa mesma direção, Weinstein et al. (2011) afirmam que conceituar estratégias de aprendizagem é uma tarefa árida, já que esse termo não tem sido utilizado de modo padronizado pelos pesquisadores (Duncan \& Mckeachie, 2005; Dunn, Lo, Mulvenon, \& Sutcliffe, 2012; Zimmerman \& Martinez-Pons, 1986). Todavia, pesquisadores convergem no que concerne às estratégias de aprendizagem envolverem aspectos cognitivos e metacognitivos, bem como que sua utilização por parte do estudante aumenta as suas chances de sucesso nas atividades escolares e acadêmicas. Atualmente, as estratégias de aprendizagem têm sido definidas como um conjunto de procedimentos que possibilitam e fortalecem a aprendizagem autorregulada (Weinstein et al., 2011). Considerando a reconhecida importância da cognição e da metacognição na conceituação das estratégias de aprendizagem, Dembo (1994) esclarece que as estratégias cognitivas se referem a um conjunto de comportamentos que influenciam o processo de aprendizagem de maneira que a informação possa ser armazenada mais eficientemente, ao passo que as metacognitivas são procedimentos que o indivíduo usa para planejar, monitorar e regular o seu próprio pensamento.

Ademais, investigações mostram que a aprendizagem de qualidade é fortemente marcada pelas emoções, tanto de forma positiva como negativa (Costa \& Boruchovitch, 2004; Cruvinel \& Boruchovitch, 2010; Pekrun, Goetz, Frenzel, Barchfeld, \& Perry, 2011). Ansiedade à prova foi a emoção mais estudada em pesquisas relacionadas à aprendizagem no contexto educativo (Costa \& Boruchovitch, 2004; Pekrun et al., 2011; Tobias, 1985). Mais recentemente, pesquisadores começam a se dedicar, de forma mais sistemática, ao estudo de diversas outras emoções também muito associadas ao contexto educativo como: alegria, raiva, tristeza, vergonha, humilhação e tédio, entre outras (Mega et al., 2013; Pekrun et al., 2011; Pekrun, Goetz, Titz, \& Perry, 2002). Preocupações passaram a ser não só acerca de quais são as emoções que mais incidem em situações de aprendizagem e como elas a afetam, mas também sobre quais estratégias os estudantes utilizam para regulá-las (Bortolleto \& Boruchovitch, 2013; Cruvinel \& Boruchovitch, 2011; Mega et al., 2013; Pekrun et al., 2011).

A autorregulação das emoções é a expressão usada para definir os processos envolvidos na forma de lidar com níveis elevados de emoções positivas e negativas. Compreende vários processos internos e externos empregados pelos indivíduos para monitorar, examinar e alterar reações decorrentes de emoções (Kopp, 1989; Gross, 1998; Thompson, 1994). Existem várias estratégias para 
regular as emoções: reavaliação cognitiva da emoção (cognitive reappraisal), a supressão da expressão da emoção (expressive suppression), o culpar a si próprio, culpar o outro, exercer controle sobre ruminações do pensamento e colocar a situação em perspectiva são alguns exemplos dessas estratégias, que podem ser mais ou menos adaptativas a depender do contexto ou situação em que são empregadas. Maiores informações acerca das estratégias de regulação da emoção podem ser encontradas em Boruchovitch e Bortolleto (2010); Kopp (1989); Gross (1998); Gross, Richards e John (2006) e Thompson (1994), entre outros.

Perassinoto (2011) investigou as estratégias de aprendizagem relatadas por alunos de $2^{a}, 4^{a}, 6^{a}$ e $8^{a}$ séries do Ensino Fundamental em relação ao gênero, idade, série e rendimento escolar. Também analisou a correlação entre as estratégias de aprendizagem e a regulação emocional e entre as estratégias de aprendizagem e o tipo de motivação para aprender dos alunos. Para tanto utilizou a Escala de Estratégias de Aprendizagem do Ensino Fundamental (EAEF), a Escala de Motivação para Aprender de Alunos do Ensino Fundamental (EMA), a Escala de Avaliação da Regulação Emocional para Estudantes do Ensino Fundamental (EREEF) e o Rendimento Escolar dos alunos nas disciplinas de Português e Matemática. Os resultados indicaram uma diferença significativa entre os gêneros no escore total da escala de estratégias de aprendizagem. Meninas mencionaram ser mais estratégicas do que os meninos, ou seja, elas relataram utilizar mais estratégias no processo de aprender. Dados como os obtidos por Perassinoto (2011) corroboram os de Carvalho (2006) e Oliveira (2008). No entanto, há estudos que não obtiveram diferenças (Cruvinel, 2003; Metallidou \& Vlachou, 2007; Mok, Ma, Lui, \& So, 2005). Na relação entre a pontuação na escala de estratégias de aprendizagem e a idade dos alunos, o estudo de Perassinoto (2011), quanto à pontuação total da escala de estratégias de aprendizagem, verificou que os alunos mais jovens obtiveram escores superiores aos dos mais velhos. Contudo, na subescala estratégias metacognitivas, os alunos maiores pontuaram mais, relatando mais repertório metacognitivo do que os mais jovens. Dados similares foram encontrados nos estudos de Carvalho (2006) e Oliveira (2008).

$\mathrm{Na}$ pontuação total da escala de estratégias de aprendizagem, os alunos da $2^{a}$ e da $4^{a}$ séries da pesquisa de Perassinoto (2011) obtiveram escores superiores aos da $8^{a}$, revelando que os alunos com menor escolarização relataram usar mais estratégias. Perassinoto (2011) destacou, ainda, a obtenção de correlações positivas entre as estratégias de aprendizagem e a regulação emocional, tendo sido observadas relações entre a pontuação na escala de estratégias e os escores nas subescalas de tristeza, raiva, medo e alegria.

Costa (2000), investigando a relação entre a ansiedade e as estratégias de aprendizagem, observou que tanto alunos com pequeno repertório de estratégias quanto aqueles que utilizam estratégias mais complexas possuíram uma 
tendência a maiores níveis de ansiedade. Cruvinel (2003) investigou a correlação entre os sintomas depressivos, as estratégias de aprendizagem e o rendimento escolar, constatando que os sintomas depressivos se associaram, de maneira negativa, ao uso das estratégias de aprendizagem e ao rendimento escolar.

A importância de se identificar as estratégias de aprendizagem e de regulação da emoção de crianças brasileiras, a necessidade de se compreender a inter-relação dos aspectos cognitivos, metacognitivos e afetivos na aprendizagem, acopladas à escassez de pesquisas na literatura nacional acerca desses aspectos conjuntamente no Ensino Fundamental (Perassinoto, 2011) motivaram a realização dessa pesquisa. Assim, o presente estudo tem como objetivos caracterizar o uso de estratégias de aprendizagem e de regulação emocional de crianças brasileiras do Ensino Fundamental, examinar se existem diferenças nessas variáveis em razão do sexo, o ano escolar e a idade dos estudantes, bem como explorar possíveis relações entre elas.

\section{MÉTODO}

\section{Participantes}

Participaram deste estudo 38 estudantes, ambos os sexos, entre 7 e 15 anos $(M=10,54 ; D P=1,90)$, do segundo ao nono ano do Ensino Fundamental de escolas públicas do Paraná. Do total da amostra, 4 escolares $(10,5 \%)$ frequentavam o segundo ano, $4(10,5 \%)$ o terceiro, $6(10,8 \%)$ o quarto, 8 $(21,1 \%)$ o quinto, $7(18,4 \%)$ o sexto, $7(18,4 \%)$ o sétimo, $1(1,6 \%)$ o oitavo e 1 $(1,6 \%)$ o nono ano. A maioria dos participantes era do sexo feminino $(n=22$; $57,9 \%)$. A seleção da amostra foi realizada por conveniência.

\section{Instrumentos}

Questões de identificação

Visaram obter dados sobre os seguintes aspectos dos participantes: nome, idade, sexo e ano escolar que frequentam. O nome foi imediatamente substituído por um número de protocolo, conforme recomendação da legislação sobre a ética na pesquisa.

Escala de Avaliação das Estratégias de Aprendizagem para o Ensino Fundamental (EAVAP-EF)

A escala foi construída por Boruchovitch e Santos, em 2001 (Boruchovitch et al., 2006) para avaliar o repertório de estratégias de aprendizagem dos alunos do Ensino Fundamental e a frequência com que relatam utilizá-las. O instrumento passou por reformulações e sua última versão, utilizada neste estudo, é composta por 31 itens, em escala Likert de três pontos (sempre, às vezes e nunca). Exemplos de questões são: "Quando você está assistindo a uma 
aula, costuma anotar o que o(a) professor(a) está falando, mesmo quando ele(a) não manda ou não escreve nada na lousa?"; "Quando você estuda, percebe se não está conseguindo aprender?" e "Você costuma ficar pensando em outra coisa quando o(a) professor(a) está dando explicações?". A opção "sempre" vale 2 pontos, às vezes, 1 , e nunca, 0 . Essa pontuação é invertida nos itens $3,7,8,12$, $15,19,21,23,24,25,26,28$ e 30 . A pontuação mínima é zero e a máxima, 62 pontos. Quanto maior é o escore obtido na escala total, maior é a tendência de que o estudante seja estratégico (Oliveira, Boruchovitch, \& Santos, 2010).

A análise fatorial indicou que os itens se agruparam em três fatores: estratégias cognitivas, estratégias metacognitivas e ausência de estratégias metacognitivas disfuncionais. A consistência interna da escala total foi de $a=0,79$ e das subescalas foi de 0,74 para estratégias cognitivas, 0,62 para estratégias metacognitivas e 0,80 para ausência de estratégias metacognitivas disfuncionais. Na presente amostra foram obtidos os seguintes índices de consistência interna: 0,74 para a escala total, 0,54 para estratégias cognitivas, 0,76 para estratégias metacognitivas e 0,58 para ausência de estratégias metacognitivas disfuncionais.

Escala de Avaliação da Regulação Emocional para Estudantes do Ensino Fundamental (ERE-EF)

Desenvolvida por Cruvinel e Boruchovitch (2008) com o objetivo de conhecer como os alunos do Ensino Fundamental lidam com suas emoções. A escala é formada por quatro subescalas: tristeza, raiva, medo e alegria. Seus itens foram construídos em forma de uma escala Likert de três pontos (sempre, às vezes e nunca), após extensa análise de conteúdo de respostas de crianças que responderam a um instrumento qualitativo sobre a regulação de suas emoções (Cruvinel \& Boruchovitch, 2010). Os itens foram redigidos em primeira pessoa e visam mapear quatro aspectos principais envolvidos na regulação emocional: a) percepção das emoções, b) motivos que as geram, c) estratégias de como lidar com elas (melhorando-as ou mantendo-as) e d) seu caráter prejudicial para o indivíduo. A única subescala para a qual não se mapeia o caráter prejudicial é alegria. A escala foi utilizada pela primeira vez na pesquisa de Perasinotto (2011), depois de ter sido pré-testada por um estudo piloto que não identificou problemas quanto à inteligibilidade dos itens.

A quantidade de itens das subescalas de tristeza, raiva, medo e alegria não é homogênea, sendo 33, 30, 33 e 17, respectivamente. Alguns exemplos de itens da escala são: "Quando estou triste, eu me distraio com outras coisas"; "Sinto raiva quando as coisas não acontecem do jeito que eu quero"; "Quando sinto medo, não consigo me concentrar"; "Eu percebo quando deixei alguém alegre". A opção sempre vale 2 pontos, às vezes, 1 , e nunca 0 . A pontuação é invertida nos itens: 9, 12, 13, 14, 15, 16, 17 e 18 (subescala de tristeza), 37, $38,42,43,45,46,48,49,50$ e 52 (subescala de raiva) e 67, 69, 70, 71, 72, 73, 75 e 76 (subescala de medo). Os valores mínimos e máximos são: de 0 a 66 
pontos para tristeza, de 0 a 60 para raiva, de 0 a 66 para medo e de 0 a 34 pontos para alegria, que perfazem um total de 226 pontos possíveis para a escala. Quanto maior é o escore obtido em cada subescala ou na escala total, melhor o sujeito lida com suas emoções e maior é o seu repertório de estratégias de Regulação Emocional.

A consistência interna da escala total estimada por Perassinoto (2011) em uma amostra de 314 estudantes de $2^{a}, 4^{a}, 6^{a}$ e $8^{a}$ séries (atuais $3^{\circ}, 5^{\circ}, 7^{0}$ e $9^{\circ}$ anos) do Ensino Fundamental foi de 0,85 e das subescalas foram: de 0,65 para tristeza, de 0,56 para raiva, de 0,65 para medo e de 0,89 para alegria. No que diz respeito à amostra do presente estudo, os índices obtidos foram: 0,94 para a escala total, 0,79 para tristeza, 0,77 para raiva, 0,86 para medo e 0,92 para alegria.

\section{Procedimentos}

\section{Procedimento de Coleta de Dados}

A coleta de dados foi realizada por meio de respostas em lápis e papel. Após a aprovação do projeto pelo Comitê de Ética (CAAE: 59601916.0.0000.5231), os instrumentos foram aplicados, individualmente, em horário de aula previamente cedido pelos professores nas escolas, que autorizaram a realização da pesquisa. Inicialmente, os estudantes, cujos pais assinaram o Termo de Consentimento Livre e Esclarecido e que concordaram em participar da pesquisa, preencheram as questões de identificação do sujeito e, em seguida, o EAVAP-EF e o ERE-EF. Em todas as aplicações, os itens dos instrumentos foram lidos um a um pela aplicadora. Para a coleta, foram utilizados régua, lápis ou caneta e borracha. O tempo de preenchimento foi, em média, de 50 minutos.

\section{Procedimento de Análise de Dados}

Para analisar as relações entre o uso das estratégias de aprendizagem, a regulação emocional e os dados demográficos dos alunos, foram utilizadas provas de estatística descritiva e inferencial paramétricas, visto que o resultado da prova de Shapiro-Wilk (W) para a análise da curva da pontuação dos escores obtidos permitiu aceitar a hipótese de normalidade para ambos os instrumentos $(\mathrm{W}(38)=0,98 ; \mathrm{p}=0,792$ para o EAVAP-EF e $\mathrm{W}(38)=0,95 ; \mathrm{p}=0,074$ para 0 ERE-EF). Sendo assim, o teste $t$ de Student foi empregado para verificar diferenças entre os estudantes em razão do sexo e dos extremos de idade. Já a Análise de Variância de um fator (ANOVA) serviu ao propósito de compará-los em função do ano escolar frequentado. Ressalta-se, entretanto, que como a quantidade de itens para cada subescala, seja para o EAVAP-EF ou para o ERE$E F$, não é homogênea, optou-se por se trabalhar aqui com pontuações ponderadas. 
Por fim, para verificar a associação linear entre variáveis, foi utilizado o coeficiente de correlação de Pearson. O nível de significância adotado para todos os testes estatísticos foi de $5 \%$.

\section{RESULTADOS}

Os estudantes avaliados obtiveram uma pontuação média 2,21 ( $D P=0,58$ ) no EAVAP-EF. A mínima foi 0,94 e a máxima 3,38. A subescala 'Estratégias Metacognitivas' foi a que apresentou a maior média e 'Estratégias Cognitivas' a menor. A Tabela 1 traz as estatísticas descritivas das pontuações obtidas nas escalas de estratégias de aprendizagem e regulação emocional empregadas.

Tabela 1.

Pontuações Obtidas na EAVAP-EF e ERE-EF ( $\mathrm{N}=38)$

\begin{tabular}{|c|c|c|c|c|}
\hline Instrumento & Mínima & Máxima & Média & DP \\
\hline $\begin{array}{c}\text { Ausência de Estratégias } \\
\text { Metacognitivas Disfuncionais }\end{array}$ & 0,38 & 2,00 & 1,24 & 0,39 \\
\hline Estratégias Cognitivas & 0,36 & 1,55 & 0,92 & 0,34 \\
\hline Estratégias Metacogn. & 0,71 & 2,71 & 1,39 & 0,38 \\
\hline EAVAP-EF & 0,94 & 3,38 & 2,21 & 0,58 \\
\hline $\begin{array}{c}\text { Estratégias para melhorar a } \\
\text { tristeza }\end{array}$ & 0,33 & 1,83 & 0,94 & 0,30 \\
\hline Caráter prejudicial da tristeza & 0,33 & 2,00 & 1,15 & 0,44 \\
\hline Percepção da tristeza & 0,00 & 2,00 & 1,53 & 0,45 \\
\hline Motivos para a tristeza & 0,40 & 1,90 & 1,20 & 0,38 \\
\hline Tristeza & 0,73 & 1,52 & 1,15 & 0,19 \\
\hline $\begin{array}{c}\text { Estratégias para melhorar a } \\
\text { raiva }\end{array}$ & 0,29 & 1,57 & 0,94 & 0,31 \\
\hline Caráter prejudicial da raiva & 0,14 & 2,00 & 1,15 & 0,44 \\
\hline Percepção da raiva & 0,40 & 2,00 & 1,44 & 0,49 \\
\hline Motivos para a raiva & 0,00 & 2,00 & 1,06 & 0,50 \\
\hline Raiva & 0,40 & 1,57 & 1,09 & 0,27 \\
\hline $\begin{array}{c}\text { Estratégias para melhorar o } \\
\text { medo }\end{array}$ & 0,20 & 1,80 & 1,01 & 0,35 \\
\hline Caráter prejudicial do medo & 0,17 & 2,00 & 1,03 & 0,51 \\
\hline Percepção do medo & 0,00 & 2,00 & 1,13 & 0,50 \\
\hline Motivos para o medo & 0,00 & 1,62 & 0,79 & 0,40 \\
\hline Medo & 0,18 & 1,64 & 0,97 & 0,33 \\
\hline $\begin{array}{c}\text { Estratégias para manter a } \\
\text { alegria }\end{array}$ & 0,00 & 2,00 & 1,54 & 0,53 \\
\hline Percepção da alegria & 0,00 & 2,00 & 1,68 & 0,47 \\
\hline Motivos para a alegria & 0,00 & 2,00 & 1,62 & 0,44 \\
\hline
\end{tabular}




\begin{tabular}{ccccc}
\hline Alegria & 0,00 & 2,00 & 1,62 & 0,44 \\
\hline ERE-EF & 0,39 & 1,60 & 1,15 & 0,25 \\
\hline
\end{tabular}

A pontuação média obtida no ERE-EF foi 1,15 ( $D P=0,25)$, com mínima de 0,39 e máxima 1,60 pontos. No que concerne às subescalas que compõem o instrumento, a maior média foi observada em alegria $(M=1,62 ; D P=0,44)$ e a menor em medo $(M=0,97 ; D P=0,33)$. Em duas das subescalas de regulação emocional, tristeza e raiva, as categorias de "Percepção da emoção' e 'Estratégias para melhorá-las' obtiveram, respectivamente as maiores e menores médias. Para as outras duas, medo e alegria, as maiores pontuações médias foram observadas em 'Percepção da emoção' e as menores em 'Estratégias para manter a alegria' e 'Motivos para o medo'.

Buscou-se, ainda, verificar se as pontuações obtidas nos instrumentos sofreriam alterações ao se considerarem variáveis como o sexo, o ano escolar e a idade dos estudantes. A Tabela 2 traz as diferenças relativas ao sexo dos participantes do estudo no EAVAP-EF.

Tabela 2.

Pontuações Obtidas no EAVAP-EF em Razão do Sexo

\begin{tabular}{ccccccc}
\hline Instrumento & Sexo & $\mathrm{N}$ & Média & $\mathrm{DP}$ & $\mathrm{t}$ & $\mathrm{P}$ \\
\hline Ausência de Estratégias & Feminino & 22 & 1,28 & 0,40 & 0,70 & 0,488 \\
Metacognitivas Disfuncionais & Masculino & 16 & 1,19 & 0,37 & & \\
& Feminino & 22 & 0,95 & 0,33 & 0,51 & 0,611 \\
Estratégias Cognitivas & Masculino & 16 & 0,89 & 0,35 & & \\
& Feminino & 22 & 1,42 & 0,43 & 0,53 & 0,596 \\
Estratégias Metacognitivas & Masculino & 16 & 1,36 & 0,32 & & \\
\hline \multirow{2}{*}{ EAVAP-EF } & Feminino & 22 & 2,27 & 0,58 & 0,77 & 0,448 \\
& Masculino & 16 & 2,12 & 0,60 & & \\
\hline \multirow{2}{*}{ Estratégias para melhorar a } & Feminino & 22 & 0,91 & 0,31 & $-0,67$ & 0,509 \\
tristeza & Masculino & 16 & 0,98 & 0,29 & & \\
& Feminino & 22 & 1,10 & 0,45 & $-0,83$ & 0,412 \\
Peráter prejudicial da tristeza & Masculino & 16 & 1,22 & 0,43 & & \\
& Feminino & 22 & 1,59 & 0,45 & 0,94 & 0,356 \\
& Masculino & 16 & 1,45 & 0,46 & & \\
Motivos para a tristeza & Feminino & 22 & 1,25 & 0,37 & 0,95 & 0,347 \\
& Masculino & 16 & 1,13 & 0,38 & & \\
\hline \multirow{2}{*}{ Tristeza } & Feminino & 22 & 1,15 & 0,20 & 0,17 & 0,862 \\
& Masculino & 16 & 1,14 & 0,19 & & \\
\hline Estratégias para melhorar a & Feminino & 22 & 0,98 & 0,26 & 0,77 & 0,451 \\
& Masculino & 16 & 0,89 & 0,38 & & \\
\hline
\end{tabular}




\begin{tabular}{|c|c|c|c|c|c|c|}
\hline \multirow{2}{*}{ Caráter prejudicial da raiva } & Feminino & 22 & 1,21 & 0,49 & 0,93 & 0,359 \\
\hline & Masculino & 16 & 1,08 & 0,36 & & \\
\hline \multirow{2}{*}{ Percepção da raiva } & Feminino & 22 & 1,44 & 0,48 & 0,12 & 0,901 \\
\hline & Masculino & 16 & 1,42 & 0,51 & & \\
\hline \multirow{2}{*}{ Motivos para a raiva } & Feminino & 22 & 1,07 & 0,52 & 0,03 & 0,973 \\
\hline & Masculino & 16 & 1,06 & 0,49 & & \\
\hline \multirow{2}{*}{ Raiva } & Feminino & 22 & 1,12 & 0,24 & 0,79 & 0,437 \\
\hline & Masculino & 16 & 1,05 & 0,31 & & \\
\hline \multirow{2}{*}{$\begin{array}{c}\text { Estratégias para melhorar o } \\
\text { medo }\end{array}$} & Feminino & 22 & 1,08 & 0,28 & 1,25 & 0,223 \\
\hline & Masculino & 16 & 0,92 & 0,42 & & \\
\hline \multirow{2}{*}{ Caráter prejudicial do medo } & Feminino & 22 & 1,11 & 0,50 & 1,13 & 0,265 \\
\hline & Masculino & 16 & 0,92 & 0,51 & & \\
\hline \multirow{2}{*}{ Percepção do medo } & Feminino & 22 & 1,28 & 0,45 & 2,31 & 0,028 \\
\hline & Masculino & 16 & 0,91 & 0,51 & & \\
\hline \multirow[t]{2}{*}{ Motivos para o medo } & Feminino & 22 & 0,87 & 0,35 & 1,42 & 0,166 \\
\hline & Masculino & 16 & 0,68 & 0,44 & & \\
\hline \multirow{2}{*}{ Medo } & Feminino & 22 & 1,06 & 0,27 & 1,93 & 0,065 \\
\hline & Masculino & 16 & 0,85 & 0,37 & & \\
\hline \multirow{2}{*}{$\begin{array}{c}\text { Estratégias para manter a } \\
\text { alegria }\end{array}$} & Feminino & 22 & 1,70 & 0,37 & 2,00 & 0,058 \\
\hline & Masculino & 16 & 1,33 & 0,65 & & \\
\hline \multirow{2}{*}{ Percepção da alegria } & Feminino & 22 & 1,74 & 0,34 & 0,81 & 0,425 \\
\hline & Masculino & 16 & 1,60 & 0,60 & & \\
\hline \multirow{2}{*}{ Motivos para a alegria } & Feminino & 22 & 1,71 & 0,28 & 1,39 & 0,179 \\
\hline & Masculino & 16 & 1,49 & 0,58 & & \\
\hline \multirow{2}{*}{ Alegria } & Feminino & 22 & 1,72 & 0,28 & 1,40 & 0,176 \\
\hline & Masculino & 16 & 1,50 & 0,58 & & \\
\hline \multirow{2}{*}{ ERE-EF } & Feminino & 22 & 1,20 & 0,19 & 1,37 & 0,183 \\
\hline & Masculino & 16 & 1,08 & 0,30 & & \\
\hline
\end{tabular}

Como pode ser observado na Tabela 2, as médias das meninas em estratégias de aprendizagem foram mais altas, quando comparadas às dos meninos em todas as situações, embora as diferenças constatadas não tenham sido estatisticamente significativas. No que concerne ao ERE-EF, os resultados indicaram que as pontuações médias femininas também foram maiores, quando comparadas às masculinas, em 17 das 19 situações analisadas. No entanto, as diferenças constatadas foram estatisticamente significativas somente na categoria 'Percepção do medo'.

Com o intuito de verificar se haveria diferença entre os estudantes ao se analisar o ano escolar por eles frequentado, recorreu-se à Análise de Variância (ANOVA). Considerando o número reduzido de participantes de cada ano escolar, 
optou-se por comparar os estudantes pensando-se no início ( $2{ }^{\circ}, 3^{\circ}$ e $4{ }^{\circ}$ ano), meio ( $5^{\circ}$ e $6^{\circ}$ ano) e final $\left(70,8^{\circ}\right.$ e $9^{\circ}$ ano) do Ensino Fundamental. A análise evidenciou diferença significativa entre a utilização de estratégias de aprendizagem e o momento do curso para 'Estratégias Cognitivas' $([\mathrm{H}(2,35)=$ $3,59 ; p=0,038]$ ) e, marginalmente significativa, para a pontuação total no 'EAVA-EF' $([\mathrm{H}(2,35)=3,19 ; \mathrm{p}=0,053])$. Em ambos os casos, o teste de Tukey evidenciou que os escolares do final do Ensino Fundamental, com a menor média de utilização de estratégias $(M=0,71$ e $M=1,81$, respectivamente), se diferenciaram dos do meio do curso, que obtiveram a maior média $(M=1,07$ e $M=2,37$, respectivamente). No caso do ERE-EF observou-se diferença estatisticamente significativa entre os estudantes do final do Ensino Fundamental, com a menor média $(M=0,69)$ e os do meio do curso, com a maior média $(M=1,03)$, somente para 'Estratégias para melhorar a raiva' $([\mathrm{H}(2,35)=$ 4,49; $p=0,018])$.

Para verificar se haveria diferença no uso de estratégias de aprendizagem e na regulação emocional também em relação à idade, recorreu-se a análise dos seus extremos. Para tanto, agrupou-se essa variável em três grupos, quais sejam, até 9 anos; de 9 a 11 anos e 12 ou mais anos. Foi utilizado o teste $t$ de Student para a comparação entre o grupo constituído pelos alunos mais novos e o formado pelos escolares mais velhos. Esses dados podem ser visualizados, juntamente com outros, na Tabela 3.

Tabela 3.

Pontuações Obtidas nos Instrumentos Empregados em Função dos Extremos de Idade

\begin{tabular}{|c|c|c|c|c|c|c|}
\hline Instrumento & $\begin{array}{l}\text { Extremos de } \\
\text { Idade }\end{array}$ & $\mathrm{N}$ & Média & $\mathrm{DP}$ & $\mathrm{t}$ & $\mathrm{p}$ \\
\hline \multirow{3}{*}{$\begin{array}{c}\text { Ausência de Estratégias } \\
\text { Metacognitivas Disfuncionais }\end{array}$} & Até 9 anos & 11 & 1,28 & 0,41 & \multirow[b]{2}{*}{1,65} & \multirow[b]{2}{*}{0,115} \\
\hline & $\begin{array}{l}12 \text { anos ou } \\
\text { mais }\end{array}$ & 11 & 1,08 & 0,39 & & \\
\hline & Até 9 anos & 11 & 0,82 & 0,27 & \multirow[b]{2}{*}{0,12} & \multirow[b]{2}{*}{0,907} \\
\hline Estratégias Cognitivas & $\begin{array}{l}12 \text { anos ou } \\
\text { mais }\end{array}$ & 11 & 0,80 & 0,38 & & \\
\hline \multirow[b]{2}{*}{ Estratégias Metacognitivas } & Até 9 anos & 11 & 1,36 & 0,37 & \multirow[b]{2}{*}{$-1,06$} & \multirow[b]{2}{*}{0,302} \\
\hline & $\begin{array}{l}12 \text { anos ou } \\
\text { mais }\end{array}$ & 11 & 1,56 & 0,48 & & \\
\hline \multirow[b]{2}{*}{ EAVAP-EF } & Até 9 anos & 11 & 2,14 & 0,47 & \multirow[b]{2}{*}{1,25} & \multirow[b]{2}{*}{0,226} \\
\hline & $\begin{array}{l}12 \text { anos ou } \\
\text { mais }\end{array}$ & 11 & 1,85 & 0,61 & & \\
\hline \multirow{2}{*}{$\begin{array}{c}\text { Estratégias para melhorar a } \\
\text { tristeza }\end{array}$} & Até 9 anos & 11 & 0,91 & 0,38 & \multirow[b]{2}{*}{0,51} & \multirow[b]{2}{*}{0,613} \\
\hline & $\begin{array}{l}12 \text { anos ou } \\
\text { mais }\end{array}$ & 11 & 0,84 & 0,23 & & \\
\hline
\end{tabular}




\begin{tabular}{|c|c|c|c|c|c|c|}
\hline \multirow[b]{2}{*}{ Caráter prejudicial da tristeza } & Até 9 anos & 11 & 0,98 & 0,41 & \multirow[b]{2}{*}{$-0,82$} & \multirow[b]{2}{*}{0,421} \\
\hline & $\begin{array}{l}12 \text { anos ou } \\
\text { mais }\end{array}$ & 11 & 1,12 & 0,36 & & \\
\hline \multirow[b]{2}{*}{ Percepção da tristeza } & Até 9 anos & 11 & 1,56 & 0,53 & \multirow[b]{2}{*}{0,19} & \multirow[b]{2}{*}{0,850} \\
\hline & $\begin{array}{l}12 \text { anos ou } \\
\text { mais }\end{array}$ & 11 & 1,53 & 0,34 & & \\
\hline \multirow[b]{2}{*}{ Motivos para a tristeza } & Até 9 anos & 11 & 1,29 & 0,38 & \multirow[b]{2}{*}{1,68} & \multirow[b]{2}{*}{0,108} \\
\hline & $\begin{array}{l}12 \text { anos ou } \\
\text { mais }\end{array}$ & 11 & 1,01 & 0,40 & & \\
\hline \multirow[b]{2}{*}{ Tristeza } & Até 9 anos & 11 & 1,14 & 0,23 & \multirow[b]{2}{*}{1,13} & \multirow[b]{2}{*}{0,276} \\
\hline & $\begin{array}{l}12 \text { anos ou } \\
\text { mais }\end{array}$ & 11 & 1,05 & 0,13 & & \\
\hline \multirow[b]{2}{*}{$\begin{array}{c}\text { Estratégias para melhorar a } \\
\text { raiva }\end{array}$} & Até 9 anos & 11 & 0,94 & 0,28 & \multirow[b]{2}{*}{1,73} & \multirow[b]{2}{*}{0,099} \\
\hline & $\begin{array}{l}12 \text { anos ou } \\
\text { mais }\end{array}$ & 11 & 0,75 & 0,22 & & \\
\hline \multirow[b]{2}{*}{ Caráter prejudicial da raiva } & Até 9 anos & 11 & 1,12 & 0,40 & \multirow[b]{2}{*}{$-0,90$} & \multirow[b]{2}{*}{0,377} \\
\hline & $\begin{array}{l}12 \text { anos ou } \\
\text { mais }\end{array}$ & 11 & 1,27 & 0,41 & & \\
\hline \multirow[b]{2}{*}{ Percepção da raiva } & Até 9 anos & 11 & 1,40 & 0,46 & \multirow[b]{2}{*}{0,34} & \multirow[b]{2}{*}{0,734} \\
\hline & $\begin{array}{l}12 \text { anos ou } \\
\text { mais }\end{array}$ & 11 & 1,33 & 0,53 & & \\
\hline \multirow[b]{2}{*}{ Motivos para a raiva } & Até 9 anos & 11 & 1,07 & 0,68 & \multirow[b]{2}{*}{0,00} & \multirow[b]{2}{*}{1,000} \\
\hline & $\begin{array}{l}12 \text { anos ou } \\
\text { mais }\end{array}$ & 11 & 1,07 & 0,35 & & \\
\hline \multirow[b]{2}{*}{ Raiva } & Até 9 anos & 11 & 1,07 & 0,25 & \multirow[b]{2}{*}{0,60} & \multirow[b]{2}{*}{0,556} \\
\hline & $\begin{array}{l}12 \text { anos ou } \\
\text { mais }\end{array}$ & 11 & 1,01 & 0,25 & & \\
\hline \multirow{2}{*}{$\begin{array}{c}\text { Estratégias para melhorar o } \\
\text { medo }\end{array}$} & Até 9 anos & 11 & 0,87 & 0,39 & \multirow[b]{2}{*}{$-0,26$} & \multirow[b]{2}{*}{0,797} \\
\hline & $\begin{array}{l}12 \text { anos ou } \\
\text { mais }\end{array}$ & 11 & 0,91 & 0,25 & & \\
\hline \multirow[b]{2}{*}{ Caráter prejudicial do medo } & Até 9 anos & 11 & 1,21 & 0,48 & & \\
\hline & $\begin{array}{l}12 \text { anos ou } \\
\text { mais }\end{array}$ & 11 & 0,77 & 0,39 & 2,35 & 0,030 \\
\hline & Até 9 anos & 11 & 1,22 & 0,41 & & \\
\hline Percepção do medo & $\begin{array}{l}12 \text { anos ou } \\
\text { mais }\end{array}$ & 11 & 1,09 & 0,54 & 0,62 & 0,542 \\
\hline & Até 9 anos & 11 & 0,80 & 0,38 & & \\
\hline Motivos para o medo & $\begin{array}{l}12 \text { anos ou } \\
\text { mais }\end{array}$ & 11 & 0,73 & 0,47 & 0,38 & 0,707 \\
\hline & Até 9 anos & 11 & 0,99 & 0,35 & & \\
\hline Medo & $\begin{array}{l}12 \text { anos ou } \\
\text { mais }\end{array}$ & 11 & 0,87 & 0,34 & 0,78 & 0,444 \\
\hline Estratégias para manter a & Até 9 anos & 11 & 1,48 & 0,46 & 0,28 & 0,779 \\
\hline
\end{tabular}




\begin{tabular}{|c|c|c|c|c|c|c|}
\hline alegria & $\begin{array}{l}12 \text { anos ou } \\
\text { mais }\end{array}$ & 11 & 1,42 & 0,54 & \multirow[b]{3}{*}{0,00} & \multirow[b]{3}{*}{1,000} \\
\hline & Até 9 anos & 11 & 1,67 & 0,30 & & \\
\hline Percepção da alegria & $\begin{array}{l}12 \text { anos ou } \\
\text { mais }\end{array}$ & 11 & 1,67 & 0,50 & & \\
\hline & Até 9 anos & 11 & 1,49 & 0,35 & \multirow[b]{2}{*}{$-0,76$} & \multirow[b]{2}{*}{0,454} \\
\hline Motivos para a alegria & $\begin{array}{l}12 \text { anos ou } \\
\text { mais }\end{array}$ & 11 & 1,63 & 0,45 & & \\
\hline \multirow[b]{2}{*}{ Alegria } & Até 9 anos & 11 & 1,54 & 0,30 & \multirow[b]{2}{*}{$-0,35$} & \multirow[b]{2}{*}{0,727} \\
\hline & $\begin{array}{l}12 \text { anos ou } \\
\text { mais }\end{array}$ & 11 & 1,60 & 0,46 & & \\
\hline \multirow[b]{2}{*}{ ERE-EF } & Até 9 anos & 11 & 1,14 & 0,23 & \multirow[b]{2}{*}{0,71} & \multirow[b]{2}{*}{0,485} \\
\hline & $\begin{array}{l}12 \text { anos ou } \\
\text { mais }\end{array}$ & 11 & 1,07 & 0,22 & & \\
\hline
\end{tabular}

Embora os participantes mais novos tenham obtido médias mais altas, quando comparados aos mais velhos, em 16 das 24 situações analisadas, verificou-se que os estudantes mais novos obtiveram uma média de pontos significativamente superior à dos mais velhos somente na categoria 'Caráter prejudicial do medo' do ERE-EF. Por fim, buscou-se verificar a relação existente entre o uso de estratégias de aprendizagem e regulação emocional. A Tabela 4 traz os índices de correlação e os níveis de significância obtidos.

Tabela 4.

Correlação de Pearson entre o Uso de Estratégias de Aprendizagem e a Regulação Emocional $(\mathrm{N}=38)$

\begin{tabular}{cccccc}
\hline Instrumento & Índice & 1 & 2 & 3 & 4 \\
\hline Estratégias para & $\mathrm{r}$ & $-0,06$ & $0,37\left(^{*}\right)$ & $-0,07$ & 0,17 \\
melhorar a tristeza & $\mathrm{p}$ & 0,699 & 0,024 & 0,688 & 0,314 \\
Caráter prejudicial & $\mathrm{r}$ & $-0,29$ & 0,02 & $-0,03$ & $-0,19$ \\
da tristeza & $\mathrm{p}$ & 0,073 & 0,912 & 0,839 & 0,264 \\
Percepção da & $\mathrm{r}$ & $-0,03$ & 0,14 & 0,32 & 0,07 \\
tristeza & $\mathrm{p}$ & 0,848 & 0,395 & 0,051 & 0,686 \\
Motivos para a & $\mathrm{r}$ & $0,88\left(^{* *}\right)$ & $0,41\left(^{*}\right)$ & $0,35\left(^{*}\right)$ & $0,83\left(^{* *}\right)$ \\
tristeza & $\mathrm{p}$ & 0,000 & 0,011 & 0,031 & 0,000 \\
\hline Tristeza & $\mathrm{r}$ & $0,35\left(^{*}\right)$ & $0,50\left(^{* *}\right)$ & 0,26 & $0,53\left(^{* *}\right)$ \\
& $\mathrm{p}$ & 0,032 & 0,001 & 0,109 & 0,001 \\
\hline Estratégias para & $\mathrm{r}$ & 0,13 & $0,44\left(^{* *}\right)$ & 0,09 & $0,34\left(^{*}\right)$ \\
melhorar a raiva & $\mathrm{p}$ & 0,444 & 0,005 & 0,598 & 0,034 \\
Caráter prejudicial & $\mathrm{r}$ & $-0,38\left(^{*}\right)$ & 0,05 & 0,27 & $-0,22$ \\
da raiva & $\mathrm{p}$ & 0,018 & 0,751 & 0,103 & 0,190 \\
\hline
\end{tabular}




\begin{tabular}{|c|c|c|c|c|c|}
\hline \multirow{2}{*}{ Percepção da raiva } & $r$ & $-0,19$ & 0,06 & 0,09 & $-0,09$ \\
\hline & $\mathrm{p}$ & 0,257 & 0,740 & 0,593 & 0,587 \\
\hline \multirow{2}{*}{ Motivos para a raiva } & $r$ & $-0,26$ & 0,02 & $-0,05$ & $-0,16$ \\
\hline & $\mathrm{p}$ & 0,121 & 0,912 & 0,774 & 0,336 \\
\hline \multirow{2}{*}{ Raiva } & $r$ & $-0,19$ & 0,28 & 0,16 & 0,04 \\
\hline & $p$ & 0,241 & 0,085 & 0,324 & 0,821 \\
\hline \multirow{2}{*}{$\begin{array}{l}\text { Estratégias para } \\
\text { melhorar o medo }\end{array}$} & $r$ & $-0,05$ & $0,33(*)$ & $-0,05$ & 0,15 \\
\hline & $\mathrm{p}$ & 0,753 & 0,044 & 0,747 & 0,353 \\
\hline \multirow{2}{*}{$\begin{array}{l}\text { Caráter prejudicial } \\
\text { do medo }\end{array}$} & $r$ & $-0,19$ & 0,15 & 0,06 & $-0,04$ \\
\hline & $\mathrm{p}$ & 0,247 & 0,371 & 0,732 & 0,812 \\
\hline \multirow{2}{*}{ Percepção do medo } & $r$ & $-0,11$ & 0,05 & 0,05 & $-0,04$ \\
\hline & $\mathrm{p}$ & 0,504 & 0,763 & 0,744 & 0,794 \\
\hline \multirow{2}{*}{ Motivos para o medo } & $r$ & $-0,15$ & 0,28 & 0,30 & 0,07 \\
\hline & $\mathrm{p}$ & 0,357 & 0,086 & 0,068 & 0,686 \\
\hline \multirow{2}{*}{ Medo } & $r$ & $-0,17$ & 0,29 & 0,15 & 0,06 \\
\hline & $\mathrm{p}$ & 0,309 & 0,074 & 0,360 & 0,718 \\
\hline \multirow{2}{*}{$\begin{array}{l}\text { Estratégias para } \\
\text { manter a alegria }\end{array}$} & $r$ & 0,04 & 0,03 & 0,01 & 0,04 \\
\hline & $\mathrm{p}$ & 0,803 & 0,859 & 0,950 & 0,787 \\
\hline \multirow{2}{*}{ Percepção da alegria } & $r$ & 0,04 & 0,07 & 0,27 & 0,07 \\
\hline & $\mathrm{p}$ & 0,802 & 0,695 & 0,097 & 0,668 \\
\hline \multirow{2}{*}{$\begin{array}{c}\text { Motivos para a } \\
\text { alegria }\end{array}$} & $r$ & 0,16 & 0,22 & 0,25 & 0,24 \\
\hline & $\mathrm{p}$ & 0,336 & 0,185 & 0,127 & 0,148 \\
\hline \multirow{2}{*}{ Alegria } & $r$ & 0,11 & 0,14 & 0,22 & 0,16 \\
\hline & $\mathrm{p}$ & 0,522 & 0,390 & 0,182 & 0,340 \\
\hline \multirow{2}{*}{ ERE-EF } & $r$ & $-0,01$ & $0,35(*)$ & 0,23 & 0,20 \\
\hline & $p$ & 0,936 & 0,032 & 0,171 & 0,232 \\
\hline
\end{tabular}

Nota. * $\mathrm{p}<0,05 ; * * \mathrm{p}<0,01 ; 1$. Ausência de Estratégias Metacognitivas Disfuncionais; 2. Estratégia Cognitiva; 3. Estratégias Metacognitivas; 4. EAVAPEF.

Como pode ser observado na Tabela 4, houve correlação estatisticamente significativa entre as medidas utilizadas em 13 das 24 situações possíveis, todas elas positivas, exceto entre 'Caráter prejudicial da raiva' e 'Ausência de Estratégias Metacognitivas Disfuncionais'. Dessas 13 associações significativas, 2 apresentaram as maiores magnitudes ('Motivos para a tristeza' e 'Ausência de Estratégias Metacognitivas Disfuncionais'; 'Motivos para a tristeza' e 'ERE-EF', respectivamente), consideradas por Dancey \& Reidy (2013) e Hemphill (2003) como fortes, 4 índices moderados ('Tristeza' e 'ERE-EF'; 'Tristeza' e 'Estratégias Cognitivas; 'Estratégias para melhorar a raiva' e 'Estratégias Cognitivas'; 'Motivos para a tristeza' e 'Estratégias Cognitivas', respectivamente) e 7 magnitudes baixas ('Estratégias para melhorar o medo' e 'Estratégias 
Cognitivas'; 'Estratégias para melhorar a raiva' e 'ERE-EF', 'Tristeza' e 'Ausência de Estratégias Metacognitivas Disfuncionais'; 'ERE-EF' e 'Estratégias Cognitivas'; 'Motivos para a tristeza' e 'Estratégias Metacognitivas'; 'Estratégias para melhorar a tristeza' e 'Estratégias Cognitivas'; 'Caráter prejudicial da raiva' e 'Ausência de Estratégias Metacognitivas Disfuncionais', respectivamente).

\section{DISCUSSÃO}

O presente estudo teve como principais objetivos caracterizar o uso de estratégias de aprendizagem e de regulação emocional de crianças brasileiras do Ensino Fundamental, examinar se existem diferenças nessas variáveis em razão do sexo, ano escolar e idade dos estudantes, bem como explorar possíveis relações entre elas. Os dados mostraram que, em relação às estratégias de aprendizagem, os estudantes da presente amostra parecem fazer uso maior das estratégias metacognitivas, tendo pouca adesão também as do tipo metacognitivas disfuncionais. As estratégias de menor média foram as cognitivas. Esses resultados são coerentes com os obtidos no estudo de Perassinoto (2011), no qual se observou esse mesmo perfil entre os 314 estudantes avaliados.

No que concerne às subescalas que compõem a escala de regulação emocional, a maior média foi observada na emoção alegria e a menor no medo. Nas subescalas, tristeza e raiva, as categorias de 'Percepção da emoção' e 'Estratégias para melhorá-las' obtiveram, respectivamente as maiores e menores médias. Felicori (2017) encontrou, em uma amostra de 108 estudantes brasileiros do Ensino Médio, que a tristeza e a raiva foram as emoções que os estudantes pareceram regular mais.

Para as outras duas, medo e alegria, as maiores pontuações médias foram observadas em 'Percepção da emoção' e as menores em 'Estratégias para manter a alegria' e 'Motivos para o medo'. Por um lado, foi interessante notar que o aspecto mais saliente da regulação emocional entre os participantes foi a percepção da emoção. Por outro, parece haver dificuldade ou desconhecimento por parte dos participantes no que tange às estratégias para lidar com as emoções, repertório esse de extrema importância, tendo em vista que a situação a ser enfrentada e o tipo de resposta emocional por ela gerada (positiva ou negativa), implicará na mobilização de diferentes estratégias que podem ser ou não adequadas e adaptativas para cada contexto (Aldao, 2013; Izard, 2010; Izard et al., 2011).

Tal como Cruvinel (2003), Metallidou e Vlachou (2007), Mok et al. (2005) e diferente de Perassinoto (2011), Carvalho (2006) e Oliveira (2008), não foram encontradas diferenças significativas entre o relato de uso de estratégias de aprendizagem e o sexo do participante. Já Felicori (2017) encontrou mais especificamente que os estudantes do sexo masculino do Ensino Médio relataram 
utilizar menos estratégias metacognitivas disfuncionais que as alunas. Considerando os resultados aqui obtidos e as inconsistências apontadas pela literatura, novos estudos que abordem esta relação e que busquem compreender melhor a associação entre as estratégias de aprendizagem e o sexo devem ser realizados. Do mesmo modo, há que se aprofundar as investigações acerca das diferenças entre meninos e meninas na regulação emocional, buscando-se identificar se, de fato, ambos percebem e lidam com as diferentes emoções da mesma forma como verificado na presente pesquisa ou se apresentam repertórios e estratégias diferenciadas para lidar com situações específicas como no caso da 'Percepção do medo', única categoria na qual se observou médias estatisticamente superiores nas meninas.

Foi instigante constatar que escolares mais avançados no curso obtiveram média significativamente menor nas estratégias do que alunos que estão no meio do curso. Aventa-se aqui a hipótese de que fatores motivacionais, embora não investigados no presente estudo, tenham mediado o relato de uso de estratégias cognitivas dos participantes. Esse dado é semelhante ao obtido por Perassinoto (2011) que investigou essas mesmas variáveis, bem como ao encontrado por Suehiro e Boruchovitch (2016), no que concerne às estratégias referentes à realização da lição de casa. Pesquisas na área da motivação para aprender mostram que ela tende a diminuir com o avanço da escolarização (Baker \& Wigfield, 1999; Gomes \& Boruchovitch, 2013; Monteiro \& Mata, 2001; Rufini, Bzuneck, \& Oliveira, 2012). Mostram também que ela pode ser uma importante variável mediadora em pesquisas relacionadas às variáveis associadas à aprendizagem (Schunk \& Zimmerman, 2008).

O ano do curso foi também uma variável que impactou significativamente somente as estratégias para melhorar a raiva. Novamente, estudantes do meio do curso mencionam lidar melhor com sua raiva do que aqueles nos anos escolares mais iniciais. Alunos mais jovens também obtiveram médias significativamente superiores quanto ao caráter prejudicial do medo. Tais achados suscitam a necessidade de novas pesquisas, já que a literatura é escassa sobre a temática e explorar a existência de diferenças de desenvolvimento relacionadas à lida com as emoções pode ter importantes implicações psicoeducacionais.

Como esperado e apontado pela literatura (Costa, 2000; Cruvinel, 2003; Perassinoto, 2011), várias foram as correlações positivas e significativas entre os aspectos cognitivos, metacognitivos e a regulação emocional dos participantes. Todavia as magnitudes foram predominantemente fracas. Sem dúvida, esse é um dado relevante na direção encontrada na literatura, sobretudo quando se leva em conta o tamanho da presente amostra. Diferentemente do observado por Perassinoto (2011), que não encontrou relações significativas entre a pontuação dos alunos na subescala estratégias cognitivas e seus escores nas subescalas de regulação emocional de tristeza, medo e alegria, no presente 
estudo houve correlações moderadas entre 'Tristeza' e 'Estratégias Cognitivas; 'Estratégias para melhorar a raiva' e 'Estratégias Cognitivas'; 'Motivos para a tristeza' e 'Estratégias Cognitivas', respectivamente.

Para além desses aspectos, a magnitude forte identificada entre 'Motivos para a tristeza' e 'Ausência de Estratégias Metacognitivas Disfuncionais' e 'Motivos para a tristeza' e 'ERE-EF', respectivamente, bem como a correlação moderada entre 'Tristeza' e 'ERE-EF' indicam que, dentre as subescalas de regulação emocional, a subescala de tristeza e seus componentes foi a que mais se destacou entre os estudantes avaliados. Pesquisas futuras devem também buscar compreender as relações negativas entre o caráter prejudicial da raiva e a ausência de estratégias metacognitivas disfuncionais.

\section{CONSIDERAÇÕES FINAIS}

A investigação acerca dos fatores cognitivos, metacognitivos e afetivos associados à aprendizagem, bem como sobre os seus possíveis entrelaçamentos é relevante e necessária, em nosso meio. Nesse sentido, o presente estudo se constituiu em uma pequena contribuição na tentativa de identificar essas variáveis e suas inter-relações. Entretanto, apresenta um conjunto de limitações que devem ser superadas pela pesquisa futura.

A literatura nacional escassa acerca das relações ora investigadas no segmento do Ensino Fundamental tornou difícil o diálogo com outras pesquisas, limitando algumas conclusões. Ademais, o número bem restrito de participantes que compuseram a amostra pode ter contribuído para a baixa consistência interna de algumas subescalas da EAVAP-EF, já que valores aceitáveis de alfa de Cronbach foram alcançados em estudos anteriores (Oliveira, 2008; Oliveira et al., 2010). Pode ainda ter afetado os resultados referentes às magnitudes das correlações encontradas.

Um outro ponto que merece destaque é a necessidade de um exame mais detalhado das propriedades psicométricas da ERE-EF em amostras maiores e mais representativas. Assim, espera-se que a presente pesquisa possa atrair novas investigações para o avanço do conhecimento referentes às complexas relações entre os componentes cognitivos e afetivos no contexto educativo.

\section{DECLARAÇÃO DE CONFLITO DE INTERESSES}

Dra. Patrícia Waltz Schelini foi editora de seção para outros artigos do número especial que compõe esta edição da revista. Dra. Evely Boruchovitch é membro do corpo editorial da Revista Estudos Interdisciplinares em Psicologia. 


\section{REFERÊNCIAS}

Aldao, A. (2013). The future of emotion regulation research: Capturing context. Perspectives on Psychological Science, 8(2), 155-172. doi: $10.1177 / 1745691612459518$.

Baker, L., \& Wigfield, A. (1999). Dimensions of children's motivation for reading and their relations to reading activity and reading achievement. Reading Research Quarterly, 34(4), 452-477. doi:10.1598/RRQ.34.4.4.

Bortoletto, D., \& Boruchovitch, E. (2013). Learning strategies and emotional regulation of pedagogy students. Paidéia, 23(55), 235-242. doi: 10.1590/1982-43272355201311.

Boruchovitch, E. (1999). Estratégias de aprendizagem e desempenho escolar: Considerações para a prática educacional. Revista Psicologia: Reflexão e Crítica, 12(2), 361-375. doi:10.1590/S0102-79721999000200008.

Boruchovitch, E., \& Bortoletto, D. (2010). Regulação emocional: Conceituação e instrumentos de medida. In A. A. A. Santos, F. F. Sisto, E. Boruchovitch, \& E. Nascimento (Eds.), Perspectivas em Avaliação Psicológica (pp. 271-292). São Paulo, SP: Casa do Psicólogo.

Boruchovitch, E., \& Santos, A. A. A. (2006). Estratégias de aprendizagem: Conceituação e avaliação. In A. P. P. Noronha, F. F. Sisto, \& A. A. A. Santos (Eds.), Facetas do fazer em avaliação psicológica (pp. 107-124). São Paulo, SP: Vetor.

Boruchovitch, E., Santos, A. A. A., Costa, E. R., Neves, E. R. C., Cruvinel, M., \& Primi, R. (2006). A construção de uma escala de estratégias de aprendizagem para alunos do Ensino Fundamental. Psicologia: Teoria e Pesquisa, 22(3), 297-304.

Boruchovitch, E., Schelini, P. W., \& Santos, A. A. A. (2010). Metacognição: Conceituação e medidas. In A. A. A. Santos, F. F. Sisto, E. Boruchovitch, \& E. Nascimento (Eds.), Perspectivas em Avaliação Psicológica (pp. 123-144). São Paulo, SP: Casa do Psicólogo.

Carvalho, M. R. (2006). Estratégias metacognitivas de leitura utilizadas de $2^{a}$ a $4^{a}$ séries do Ensino Fundamental. (Dissertação de Mestrado). Universidade São Francisco, Itatiba- SP.

Costa, E. R. (2000). As estratégias de aprendizagem e a ansiedade de alunos do Ensino Fundamental: implicações para a prática educacional. (Dissertação de Mestrado). Universidade Estadual de Campinas, Campinas- SP.

Costa, E. R, \& Boruchovitch, E (2004). Compreendendo as relações entre estratégias de aprendizagem e ansiedade de alunos do ensino fundamental de Campinas. Revista Psicologia Reflexão e Crítica, 17(1), 15-24. doi: 10.1590/S0102-79722004000100004. 
Cruvinel, M. (2003). Depressão infantil, rendimento escolar e estratégias de aprendizagem em alunos do Ensino Fundamental. (Dissertação de Mestrado). Universidade Estadual de Campinas, Campinas- SP.

Cruvinel, M, \& Boruchovitch, E. (2011). Regulação emocional em crianças com e sem sintomas de depressão. Estudos de Psicologia, 16(3), 219-226. doi: 10.1590/S1413-294X2011000300003.

Cruvinel, M., \& Boruchovitch, E. (2010). Regulação Emocional de Estudantes: A construção de um instrumento qualitativo. Psicologia em Estudo (Maringá), 15(2), 537-545. doi:10.1590/S1413-73722010000300011.

Cruvinel, M., \& Boruchovitch, E. (2008). Escala de Avaliação da Regulação Emocional para Estudantes do Ensino Fundamental - ERE-EF. Manuscrito não publicado. Universidade Estadual de Campinas, São Paulo.

Dancey, C. P., \& Reidy, J. (2013). Estatística sem matemática para psicologia. 5a. ed. Porto Alegre, RS: Artmed.

Dansereau, D. F., Collins, K. W., MacDonald, B. A., Holley, C. D., Garland, J. C. Diekhoff, G. M., \& Evans, S. H. (1979). Development and evaluation of an effective learning strategy. Journal of Educational Psychology, 79, 64-73.

Dembo, M. H. (1994). Applying educational psychology. New York, NY: Longman Publishing Group.

Duncan, T. G., \& McKeachie, W. J. (2005). The making of the motivated strategies for learning questionnaire. Educational Psychologist, 40(2), 117128. doi:10.1207/s15326985ep4002_6.

Dunn, K. E., Lo, W.-J., Mulvenon, S. W., \& Sutcliffe, R. (2012). Revisiting the motivated strategies for learning questionnaire: A theoretical and statistical reevaluation of the metacognitive self-regulation and effort regulation subscales. Educational Psychological Measurement, 72(2), 312-331. doi: $10.1177 / 0013164411413461$

Felicori, C. M. (2017). Estratégias de aprendizagem, compreensão leitora e regulação emocional: Relações com o sucesso de estudantes no ingresso no Ensino Médio. (Dissertação de Mestrado). Universidade Estadual de Campinas, Campinas- SP.

Garner, R., \& Alexander, P. A. (1989). Metacognition: Answered and unanswered questions. Educational Psychologist, 24(2), 143-158. doi:101207/sl15326985ep2402_2.

Gomes, M., \& Boruchovitch, E. (2013). Motivação para ler e compreensão leitora no Ensino Fundamental-alguns fatores relacionados. In XI CONPE Congresso Nacional de Psicologia Escolar e Educacional (pp. 3-3). Uberlândia, MG: Universidade Federal de Uberlândia.

Gross, J. J. (1998). The emerging field of emotion regulation: An integrative review. Review of General Psychology, 2(3), 271-299. doi:101037/10892680.2.3.271. 
Gross, J. J., Richards, \& J. M., John, O. P. (2006). Emotion regulation in everyday life. In D. K. Snyder, J. Simpson, \& J. N. Hughes (Eds.), Emotion regulation in couples and families: Pathways to dysfunction and health (pp. 13-35). Washington, DC, US: American Psychological Association.

Hemphill, J. F. (2003). Interpreting the magnitudes of correlation coefficients. American Psychologist, 58(1), 78-79. doi:1037/0003-066X.58.1.78.

Izard, C. E. (2010). The many meanings/aspects of emotion: Definitions, functions, activation, and regulation. Emotion Review, 2(4), 363-370. doi: $10.1177 / 1754073910374661$.

Izard, C. E., Woodbum, E. M., Finlon, K. J., Krauthamer-Ewing, E. S., Grossman, S. R., \& Seidenfeld, A. (2011). Emotion knowledge, emotion utilization, and emotion regulation. Emotion Review, 3(1), 44-52. doi: $10.1177 / 1754073910380972$.

Kopp, C. B. (1989). Regulation of distress and negative emotions: A developmental view. Developmental Psychology, 25(3), 343-354.

Linnenbrink-Garcia, L., \& Pekrun, R. (2011). Students' emotions and academic engagement: Introduction to the special issue. Contemporary Educational Psychology, 36, 1-3. doi:10.1016/j.cedpsych.2010.11.004.

Mega, C., Ronconi, L., \& De Beni, R. (2013). What makes a good student? How emotions, self-regulated learning and motivation contribute to academic achievement? Journal of Educational Psychology, 1, 1-11.

Metallidou, P., \& Vlachou, A. (2007). Motivational beliefs, cognitive engagement, and achievement in language and mathematics in elementary school children. International Journal of Psychology, 42(1), 2-15. doi:10.1080/00207590500411179.

Mok, M. M. C., Ma, H. S., Lui, F. Y. F., \& So, E. Y. P. (2005). Multilevel analysis of primary students' perception and deployment of self-learning strategies. Educational Psychology, 25(1), 129-148.

Monteiro, V., \& Mata, L. (2001). Motivação para a leitura em crianças do 10, 20 , $3^{\circ}$ e 4 anos de escolaridade. Infância e Educação - Investigação e Práticas, 3, 49-68.

Nisbett, J., \& Schucksmith, J. (1986). Learning Strategies. Londres, UK: Routledge and Keagan Paul.

Oliveira, K. L. (2008). Escala de estratégias de aprendizagem para o Ensino Fundamental: análise de suas propriedades psicométricas. (Tese de Doutorado). Universidade Estadual de Campinas, Campinas- SP.

Oliveira, K. L., Boruchovitch, E., \& Santos, A. A. A. (2010). Escala de avaliação de estratégias de aprendizagem para o ensino fundamental - EAVAP - EF Manual. São Paulo, SP: Casa do Psicólogo. 
Pekrun, R., Goetz, T., Frenzel, A. C., Barchfeld, P., \& Perry, R. P. (2011). Measuring emotions in students' learning and performance: The Achievement Emotions Questionnaire (AEQ). Contemporary Educational Psychology, 36, 36-48. doi:10.1016/j.cedpsych.2010.10.002.

Pekrun, R., Goetz, T., Titz, W., \& Perry, R. P. (2002). Academic emotions in students' self-regulated learning and achievement: A program of qualitative and quantitative research. Educational Psychologist, 37, 91-105. doi: $10.1207 /$ S15326985EP3702_4.

Perassinoto, M. G. M. (2011). Estratégias de Aprendizagem no Ensino Fundamental: Relações com Regulação Emocional, Motivação e Rendimento Escolar. (Dissertação de Mestrado). Universidade Estadual de Campinas, Campinas- SP.

Pozo, J. I. (1996). Estratégias de Aprendizagem. In C. Coll, J. Palácios, \& A. Marchesi (Eds.), Desenvolvimento psicológico e educação: Psicologia da educação (pp. 176-197). Porto Alegre, RS: Artes Médicas.

Pressley, M., Borkowski, J. G., \& Schneider, W. (1989). Good information processing: What it is and how education can promote it. Journal of Educational Research, 13(8), 857-867.

Rufini, S. E., Bzuneck, J. A., \& Oliveira, K. L. (2012). Qualidade da Motivação em Estudantes do Ensino Fundamental. Paidéia, 22(51), 53-62. doi: $10.1590 /$ S0103-863X2012000100007.

Schunk, D. H., \& Zimmerman, B. J. (2008). Motivation and self-regulated learning. Theory, research and applications. New York, NY: Lawrence Erlbaum Associates.

Suehiro, A. C. B., \& Boruchovitch, E. (2016). Hábitos e estratégias para a realização da lição de casa e variáveis demográficas em estudantes do Ensino Fundamental II. Psico (Porto Alegre), 47(2), 132-139. doi: 10.15448/1980-8623.2016.2.22729.

Thompson, R. A. (1994). Emotion regulation: A theme in search of a definition. Monographs for the Society for Research in Child Development, 59, 25-52.

Tobias, S. (1985). Test anxiety: interference, defective skills, and cognitive capacity. Educational Psychologist, 20(3), 133-142. doi: 10.1207/s15326985ep2003_3.

Weinstein, C. E., Acee, T. W., \& Jung, J. (2011). Self-regulation and learning strategies. New Directions for Teaching and Learning, 126, 45-53. doi: $10.1002 /$ tl.443.

Weinstein, C. E., \& Mayer, R. E. (1985). The teaching of learning strategies. In M. Wittrock (Ed.), Handbook of research on teaching (p.315-327). New York, NY: Macmillan. 
Zimmerman, B. J., \& Martinez-Pons, M. (1986). Development of a structured interview for assessing student use of self-regulated learning strategies. American Educational Research Journal, 23(4), 614-628. doi: $10.3102 / 00028312023004614$.

Zimmerman, B. J., \& Schunk, D. H. (2011). Self-regulated learning and performance: an introduction and an overview. In B. J. Zimmerman, \& D. H. Schunk (Eds.), Handbook of self-regulation of learning and performance (pp-1-12). New York, NY: Routledge Taylor \& Francis Group.

\section{Sobre os autores}

Adriana Cristina Boulhoça Suehiro é psicóloga pela Universidade São Francisco, mestre e doutora em Psicologia pela Universidade São Francisco e pós-doutora pela Universidade Estadual de Campinas. É docente do Centro de Ciências da Saúde da Universidade Federal do Recôncavo da Bahia - Santo Antônio de Jesus. dricbs@yahoo.com.br

Evely Boruchovitch é psicóloga pela Universidade do Estado do Rio de Janeiro, Ph.D em Educação pela University of Southern Califórnia, Los Angeles. É Professora Titular do Departamento de Psicologia Educacional e do Programa de Pós-Graduação em Educação da Universidade Estadual de Campinas. Bolsista Produtividade em Pesquisa do CNPq 1B. evely@unicamp.br Patrícia Waltz Schelini é psicóloga pela Pontifícia Universidade Católica de Campinas, mestre e doutora em Psicologia pela Pontifícia Universidade Católica de Campinas, pós-doutora pela Universidade do Minho. É docente pesquisadora do curso de Pós-Graduação Stricto Sensu em Psicologia pela Universidade Federal de São Carlos. Patriciaws01@gmail.com

A contribuição de cada autora pode ser atribuída como se segue: A.C.B.S. contribuiu com a execução de todas as etapas do estudo e, especialmente, com a descrição e análise dos resultados, bem como com a elaboração e revisão do texto final. E.B. e P.W.S. contribuíram com a redação da introdução, da revisão de literatura, bem como com a redação da definição dos conceitos-chave, revisão das análises e redação da discussão do artigo; A.C.B.S., E.B. e P.W.S. foram as responsáveis pela redação final (revisão e edição).

Recebido em: 25/09/2017

Revisado em: 08/06/2018

Aceito em: $16 / 06 / 2018$ 